\title{
Expression and histopathological correlation of CCR9 and CCL25 in ovarian cancer
}

\author{
RAJESH SINGH ${ }^{1}$, CECIL R. STOCKARD ${ }^{2}$, WILLIAM E. GRIZZLE ${ }^{2}$, \\ JAMES W. LILLARD $\mathrm{Jr}^{1}$ and SHAILESH SINGH ${ }^{1}$ \\ ${ }^{1}$ Department of Microbiology, Biochemistry and Immunology, Morehouse School of Medicine, Atlanta, \\ GA 30310; ${ }^{2}$ Department of Pathology, University of Alabama at Birmingham, Birmingham, AL 35294, USA
}

Received February 21, 2011; Accepted March 30, 2011

DOI: 10.3892/ijo.2011.1059

\begin{abstract}
Ovarian carcinoma is the most lethal gynecological malignancy among women and its poor prognosis is mainly due to metastasis. Chemokine receptor CCR9 is primarily expressed by a small subset of immune cells. The interactions between CCL25 and CCR9 have been implicated in leukocyte trafficking to the small bowel, a frequent metastatic site for ovarian cancer cells. We have previously shown that ovarian cancer cells express CCR9 and play an important role in cell migration, invasion and survival in the presence of its natural ligand in vitro. In this study, we have evaluated the expression of CCR9 and CCL25 in ovarian cancer cells and clinical samples. Ovarian cancer tissue microarrays from University of Alabama at Birmingham and AccuMax were stained for CCR9 and CCL25. Aperio ScanScope was used to acquire 80X digital images and expression analysis of CCR9 and CCL25. Flow cytometry and the Image stream system were used to conform the expression of CCR9 and CCL25 in ovarian cancer cells. Our results show significantly higher $(p<0.001)$ expression of CCR9 and CCL25 in serous adenocarcinoma followed by serous papillary cystadenoma, endometrioid adenocarcinoma, mucinous adenocarcinoma, cystadenoma, mucinous boderline adenocarcinoma, clear cell carcinoma, granulosa cell tumor, dysgerminoma, transitional cell carcinoma, Brenner tumor, yolk sac tumor, adenocarcinoma and fibroma cases, compared to non-neoplastic ovarian tissue. Similar to tissue expression, CCR9 was also significantly expressed by the ovarian cancer cell lines (OVCAR-3 and SK-OV-3) in comparison to normal adult ovarian epithelial cell. We provide the first evidence that CCR9 and its natural ligand CCL25 are highly expressed by ovarian cancer tissue and their expression correlates with histological subtypes. Expression of this chemokine receptor and its ligand CCL25 within primary tumor tissue
\end{abstract}

Correspondence to: Dr Shailesh Singh, Department of Microbiology, Biochemistry and Immunology, Morehouse School of Medicine, 720 Westview Drive, Atlanta, GA 30310, USA

E-mail: shsingh@msm.edu

Key words: chemokine, ovarian cancer, $\mathrm{CCR} 9$ and $\mathrm{CCl} 25$ further suggests a potential role of this chemokine-receptor axis in ovarian cancer progression.

\section{Introduction}

Ovarian cancer is the fifth most common cause of death from all cancers occurring in women and is a leading cause of mortality from gynecologic cancer. Human epithelial ovarian cancer (EOC) constitutes approximately $90 \%$ of ovarian malignancies and is most thought to arise from ovarian surface epithelium (1). Despite aggressive treatment, 5-year survival rate remains only $25 \%$, the main cause of treatment failure and death for cancer patients is metastasis (2). Metastasis is the result of several sequential steps and represents a highly organized, nonrandom and organ selective process (3). Ovarian cancer is characterized by early spreading to peritoneal wall, diaphragm and omental structures. Although a number of molecules have been implicated in the metastasis, the precise mechanisms of the directional migration and invasion of tumor cells into specific organs remain to be established. Many studies suggested the mechanisms used for homing of leukocytes and hematopoietic progenitors may be appropriated for the dissemination of tumors via the bloodstream and lymphatics.

Chemokines belong to 8-10 kDa chemoattractive cytokines. They are divided into four subfamilies: $\mathrm{CC}, \mathrm{CXC}, \mathrm{C}$ and $\mathrm{CX} 3 \mathrm{C}$, on the basis of the position of their NH2-terminal cysteine residues. They possess a wide range of biological activities, including the regulation of leukocyte trafficking, the modulation of hematopoietic cell proliferation, adhesion to extracellular matrix molecules and directional migration by binding to transmembrane G-protein-coupled receptors of target cells $(4,5)$. Thymus-expressed chemokine (TECK) or CCL25, a CC chemokine expressed predominantly in thymus and epithelium of the small intestine and a ligand of CCR9, mediates chemotaxis of CCR9-bearing T cells (6,7). A number of studies have shown selective expression of CCR9 on small intestinal infiltrating $\mathrm{T}$ cells (8-10). Recent studies have also shown more evidence of this site-specific immunity by demonstrating that, in patients with inflammatory bowel disease, there are increased numbers of CCR9 (+) lymphocytes circulating in peripheral blood (11). We have previously shown the CCR9 expressed by prostate cancer cells and tissues (12) and recently show that CC chemokine receptor 9 (CCR9) and its natural ligand CCL25 
interaction up-regulates anti-apoptotic proteins (i.e. PI3K, AKT, ERK1/2 and GSK-3 $\beta$ ) and down-regulates activation of caspase-3 in prostate cancer cells (13). Based on this information, we investigated the specific expression of CCR9-CCL25 axis in the ovarian cancer. The aim of the present study was to evaluate expression of CCR9 and CCL25 in normal ovarian surface epithelium and epithelial ovarian cancer tissue by immuno-histochemical staining. We further explored the potential association between expression of CCR9 and CCL25 and clinico-pathological features.

\section{Materials and methods}

Tissue specimens. The tissue microarray (TMA) slides containing malignant and non-neoplastic ovarian tissues $(\mathrm{n}=94)$ were procured from AccuMax Array (ISU Abxis Co., Ltd.). To construct tissue microarrays, at least two cores $(1 \mathrm{~mm}$ in diameter) per patient were arrayed on a receiver blank paraffin block. A qualified pathologist concerning the histopathology, the class and the grade of the tumor validated each core of the tissue microarray one additional time. The ovarian cancer tissue microarray used in this study was composed of tumors from 43 patients. These ovarian tumors represent all histo-pathological subtypes. TMA consisted of 8 non-neoplastic, 18 serous adenocarcinoma, 2 serous papillary cystadenoma, 10 endometrioid adenocarcinoma, 4 mucinous adenocarcinoma, 6 cystadenoma, 2 mucinous boderline adenocarcinoma, 10 clear cell carcinoma, 6 granulosa cell tumor, 6 dysgerminoma, 6 transitional cell carcinoma, 2 Brenner tumor, 8 yolk sac tumor, 2 adenocarcinoma and 4 fibroma tissue samples. These tissues represented each tumor grade of ovarian cancer.

Immunohistochemistry. The TMA slides were stained with anti-human CCR9 and CCL25 mAb by antigen retrieval immunohistochemistry technique using a Vector $\mathrm{ABC}$ kit (Vector Laboratories). Briefly, the TMA slides were deparaffinized, rehydrated, and incubated in $0.3 \% \mathrm{H}_{2} \mathrm{O}_{2}$. After antigen retrieval, slides were incubated in Vectastain normal serum. The samples were then probed with anti-human CCR9 (Clone) and CCL25 mAb. After washing with PBS containing 0.05\% Tween-20 (PBS-T), samples were incubated with secondary antibody and washed again with PBS-T before incubation with $\mathrm{ABC}$ solution. The immunohistochemical reaction color was developed with 3,3'-diaminobenzidine (DAB) substrate (DAB substrate kit, Vector Laboratories). The slides were washed, counterstained with haematoxylin, dehydrated, and mounted with Vectamount permanent mounting media (Vector Laboratories). All slides were analyzed using Aperio ScanScope scanning system (Aperio Technologies).

Quantitation of immunohistochemical staining. To numerically analyze the immunohistochemical staining, virtual slides were created from the stained samples after scanning each specimen using Aperio ScanScope scanning system (Aperio Technologies). The ScanScope generated true color digital images of each stained sample, which were viewed using Aperio ImageScope (version 6.25) software. The algorithm for determining the intensity of membrane-specific staining was used to calculate the staining intensity and percent target labeled for each sample by digitally analyzing the color intensity. A color markup image for each slide was obtained based on membrane staining intensity. The output was viewed as determinations of staining intensity ranging from 0 to 3 to correlate with conventional manual scoring methods $(0$, negative; and 3, strong staining) and statistical analyses were performed using the mean of these values. Further, hierarchical cluster analysis of immunohistochemistry was performed using EPCLUST (http://www.bioinf.ebc.ee/EP/EP/EPCLUST/ index).

Cell culture. Human ovarian cancer cell lines (OVCAR-3 and SK-OV-3) were obtained from the ATCC. The cells were cultured in RPMI-1640 (Mediatech, Inc.), supplemented with $10 \%$ fetal bovine serum (FBS, Sigma) at $37^{\circ} \mathrm{C}$ with $5 \%$ $\mathrm{CO}_{2}$. Prior to each experiment, cells were cultured for $24 \mathrm{~h}$ in RPMI-1640 and 2\% charcoal-striped FBS.

Flow cytometry analysis of CCR 9 surface expression. Fluorescein-conjugated mouse anti-human CCR9 (clone 112509) antibody and fluorescein-conjugated mouse IgG2a immunoglobulin isotype control was purchased from R\&D Systems. Ovarian cancer cells were washed three times in phosphate buffered saline (PBS) [supplemented with $1 \%$ bovine serum albumin (BSA)] and treated with $1.0 \mu \mathrm{g}$ of Fc Block (Pharmingen) per $10^{5}$ cells for $15 \mathrm{~min}$ at room temperature. Fc-blocked cells were stained with $1.0 \mu \mathrm{g}$ of fluorescein-conjugated mouse anti-human CCR9 or fluorescein-conjugated mouse IgG2a isotype control antibody per $10^{5}$ cells at $4^{\circ} \mathrm{C}$ for $1 \mathrm{~h}$. Subsequently, the cells were washed with $1.0 \mathrm{ml}$ of fluorescence-activated cell-sorting (FACS) buffer (1\% BSA in PBS) to remove unbound antibodies. Next, labeled cells were fixed in $500 \mu \mathrm{l}$ of $2 \%$ paraformaldehyde solution, and $10^{5}$ cells were analyzed by flow cytometry using a FACScan flow cytometer as well as using Amnis Image stream system and data were analyzed using CellQuest software (BD Pharmingen).

Statistical analyses. CCR9 and CCL25 expression intensity by ovarian TMAs was tested for normality assumptions using the Shapiro-Wilk test and was transformed to a log scale. The general linear models (GLM) procedure was used to test the association of CCR9 and CCL25 expression and disease condition using SAS version 9.1.3 statistical analysis software. Results were declared significant at a $\alpha$ level of 0.001 . The experimental data were compared using a two-tailed Student's t-test and expressed as the mean \pm SEM. The results were analyzed using the StatView II program (Abacus Concepts, Inc.) and were recorded statistically significant at p-value $<0.01$. Using the Cell Quest Software, the Kolmogorov-Smirnov (K-S) two-sample test was used to calculate the statistical significance of the CCR9 histograms.

\section{Results}

Ovarian cancer TMAs consisting of tissue from 43 cases were evaluated for CCR 9 and CCL25 expression. Forty-three patients were diagnosed with histological subtypes, 9 serous adenocarcinoma, 1 serous papillary cystadenoma, 5 endometrioid adenocarcinoma, 2 mucinous adenocarcinoma, 3 cystadenoma, 1 mucinous boderline adenocarcinoma, 5 clear cell carcinoma, 3 granulosa cell tumor, 3 dysgerminoma, 3 transitional cell 
[A]

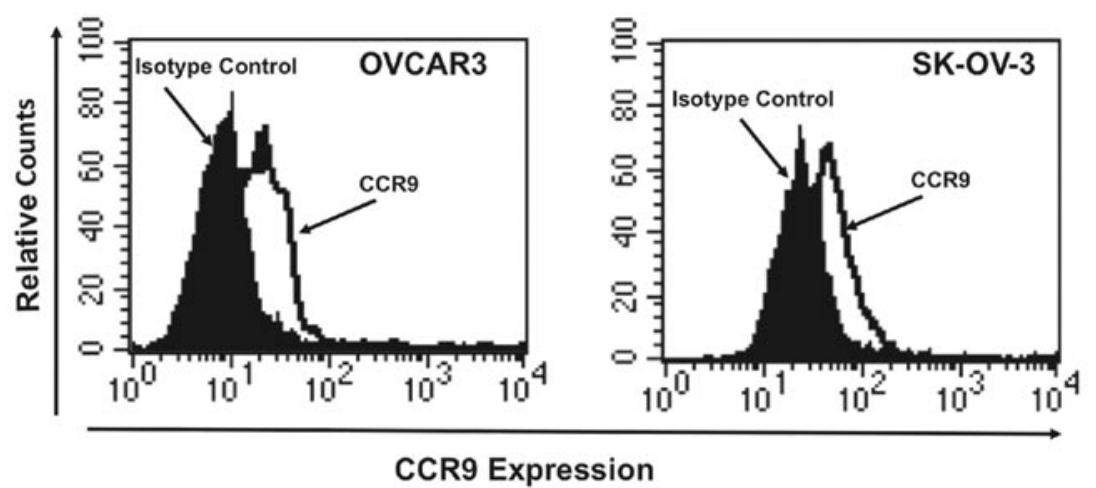

OVCAR-3 $\quad[B] \quad$ SK-OV-3

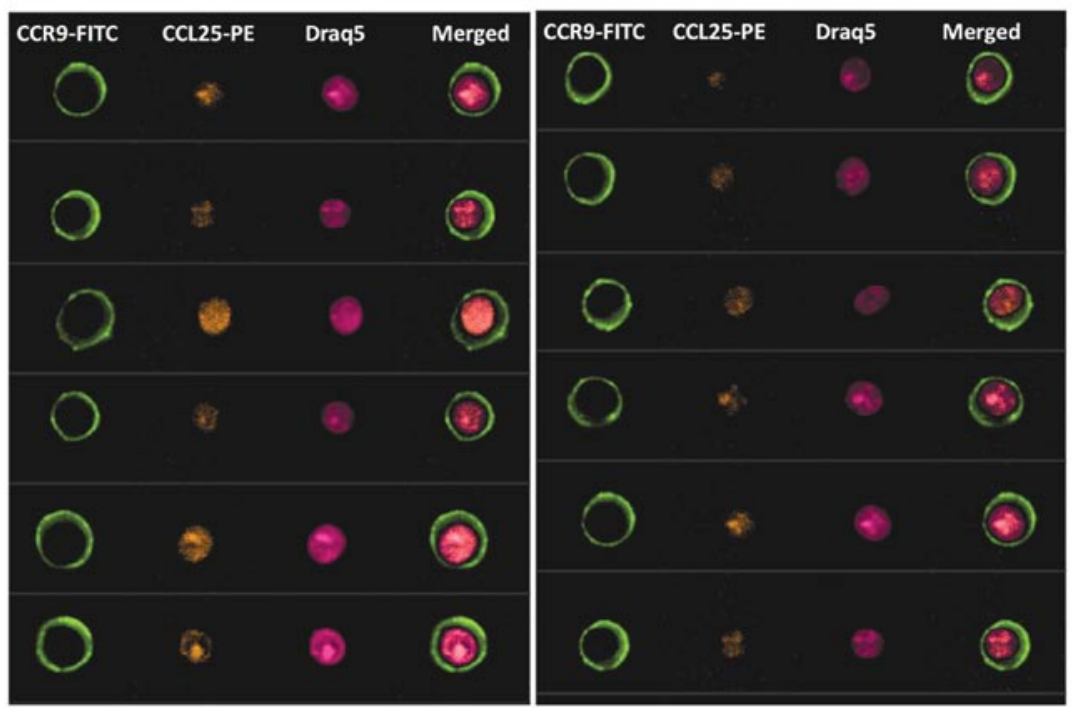

Figure 1. CCR9 and CCL25 expressed by the ovarian cancer cells (A). Ovarian cancer cells were stained with fluorescein-conjugated anti-CCR9 and fluorescein-conjugated isotype control antibody and analyzed by FACS. (B) Ovarian cancer cells were stained with fluorescein-conjugated anti-CCR9. Intracellular CCL25 was stained with PE-conjugated anti-CCL25 and nuclei were stained with Draq-5. Merged data show the expression of CCR9 on the surface and CCL25 expression in the nucleus.

carcinoma, 1 Brenner tumor, 4 yolk sac tumor, 1 adenocarcinoma and 2 fibroma. There are 8 non-neoplastic cases. CCR 9 and CCL25 expression was not detected significantly in non-neoplastic tissue (Fig. 2), but they were expressed in different ovarian carcinomas. CCR9 surface protein expression was evaluated in ovarian cancer cells by flow cytometry (Fig. 1A) and further evaluated using Amnis Image stream system (Flow-based Imaging system) to show CCR9 and CCL25 expression (Fig. 1B). To study the coordinated expression of CCR9 and CCL25 we analyzed 92-stained core of tissue samples using unsupervised hierarchical clustering analysis (average linkage method) with CCL25 and CCR9 co-expression in ovarian carcinomas (Fig. 3). All cancer specimens showed significantly $(\mathrm{p}<0.0001)$ higher expression of CCR9 and CCL25 in comparison to non-neoplastic tissue (Figs. 4 and 5). Serous adenocarcinoma significantly correlated with dysgerminoma $(\mathrm{p}=0.0023)$, yolk sac tumor $(\mathrm{p}=0.0123)$ and adenocarcinoma $(\mathrm{p}=0.0025)$ in CCR9 expression, whereas CCL25 was positively correlated with cystadenoma $(\mathrm{p}=0.0283)$, dysgerminoma $(p=0.0014)$, transitional cell carcinoma $(p=0.0092)$ and fibroma $(p=0.0044)$. Endometrioid carcinoma significantly expressed
CCL25 correlating to mucinous adenocarcinoma ( $\mathrm{p}=0.0052)$, dysgerminoma $(\mathrm{p}=0.0249)$, transitional cell carcinoma (0.0019), adenocarcinoma $(0.0438)$ and fibroma $(p=0.0337)$, whereas CCR9 expression only significantly correlated with dysgerminoma $(\mathrm{p}=0.0251)$ and adenocarcinoma $(\mathrm{p}=0.0009)$. Mucinous adenocarcinoma expressed CCR9 and CCL25 that is significantly correlated with cystadenoma $(\mathrm{p}=0.0059$ and 0.0031$)$, dysgerminoma $(\mathrm{p}=0.0026$ and 0.0002$)$, Brenner tumor $(\mathrm{p}=0.0106$ and 0.0082$)$, yolk sac tumor $(\mathrm{p}=0.0136$ and 0.0162$)$ and fibroma $(p=0.0219$ and 0.0003$)$, respectively. CCR9 expression was also higher in adenocarcinoma $(\mathrm{p}=0.0163)$ and CCL25 in mucinous boderline adenocarcinoma $(\mathrm{p}=0.0169)$ and clear cell carcinoma $(\mathrm{p}=0.0036)$. Cystadenoma expressed CCR9 as well as CCL25 that showed significant differences with transitional cell carcinoma $(\mathrm{p}=0.0451$ and 0.0021$)$ and adenocarcinoma ( $\mathrm{p}=0.0002$ and 0.02 ). Expression of CCR9 and CCL25 by mucinous boderline adenocarcinoma showed significant difference with dysgerminoma $(\mathrm{p}=0.047)$, Brenner tumor $(p=0.0052)$, transitional cell carcinoma $(p=0.044)$ and adenocarcinoma $(\mathrm{p}=0.0088$ and 0.0235$)$, similarly, clear cell carcinoma expressed the same, but granulosa cell tumor, yolk 


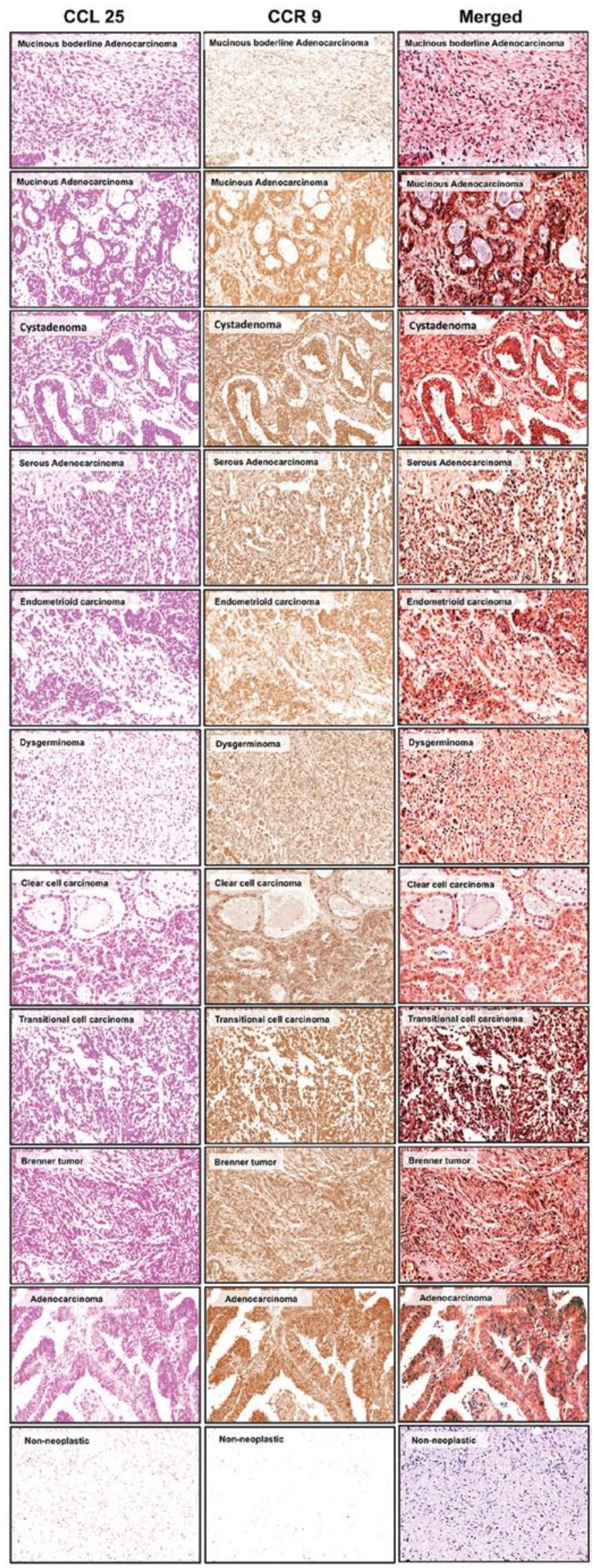

Figure 2. CCR9 and CCL25 expressed by the ovarian cancer tissue. Ovarian cancer tissues from non-neoplastic $(n=8)$, serous adenocarcinoma ( $\mathrm{n}=9$ ), serous papillary cystadenoma $(n=1)$, endometrioid adenocarcinoma $(n=5)$, mucinous adenocarcinoma $(n=2)$, cystadenoma $(n=3)$, mucinous boderline adenocarcinoma $(n=1)$, clear cell carcinoma $(n=5)$, granulosa cell tumor $(n=3)$, dysgerminoma $(n=3)$, transitional cell carcinoma $(n=3)$, Brenner tumor $(n=1)$, yolk sac tumor $(n=4)$, adenocarcinoma $(n=1)$ and fibroma $(n=2)$ were stained with isotype control or anti-CCR9 and CCL25 antibodies. Brown (DAB) color shows CCR9 staining and Magenta color show CCL25. An Aperio Scan Scope system with a x40 objective captured digital images of each slide. Representative cases are indicated and immuno-intensities of CCR9 and CCL25 were quantified using image analysis Aperio ImageScope v.6.25 software. 

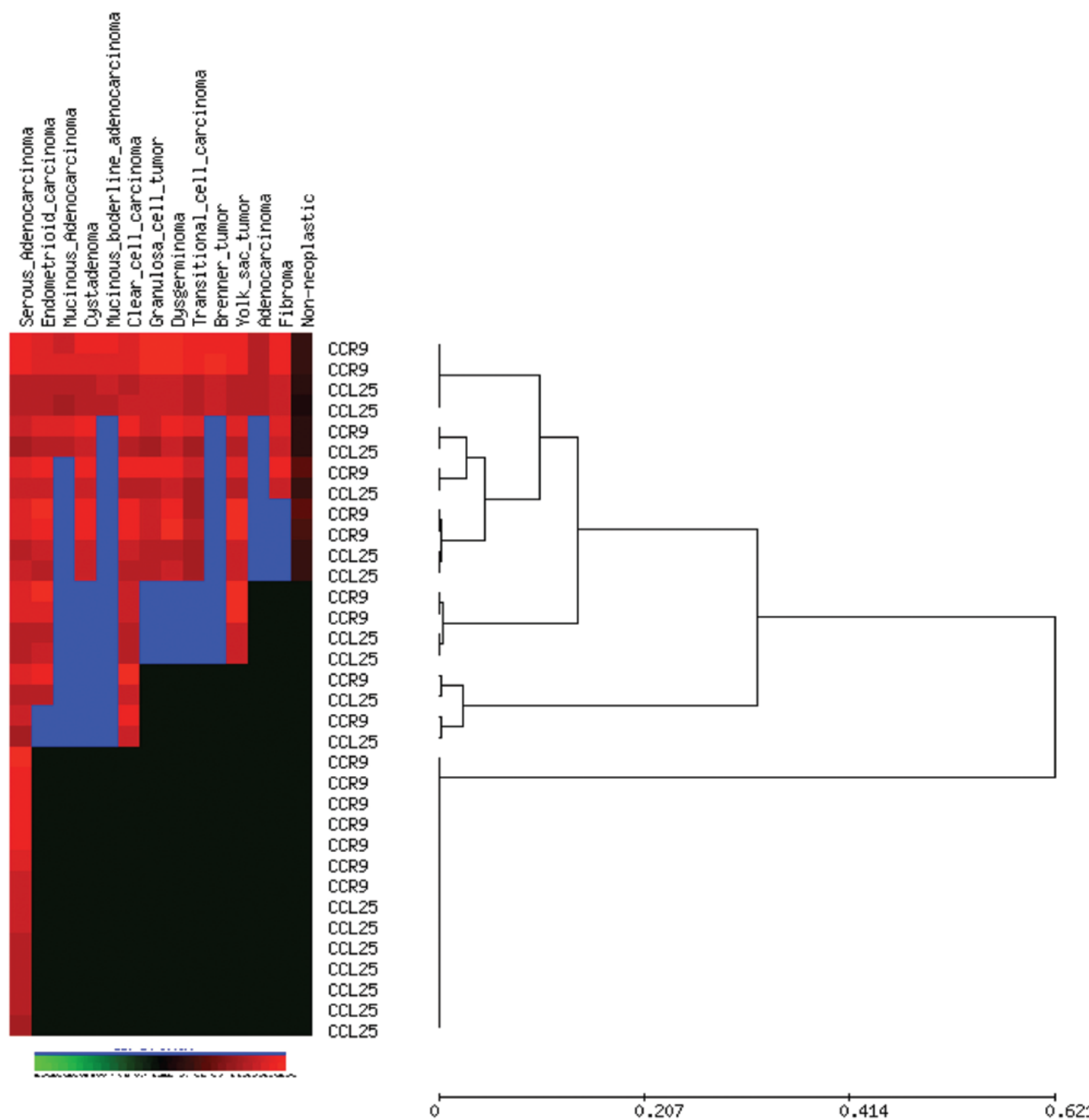

Figure 3. Hierarchial cluster analysis of CCR9 and CCL25 expression in ovarian cancer. Each column represents a different tumor and each row a different marker. The red segment of the dendogram indicates a group of cases positive for all markers with interpretable data. Green, score 0; black, score 1; brown, score 2; red, score 3; and blue, missing data.

sac tumor and fibroma was not significantly correlated with any carcinoma except transitional cell carcinoma $(\mathrm{p}=0.015)$. Transitional cell carcinoma expressed CCR9 and CCL25, which significantly correlated with Brenner tumor and yolk sac tumor. These results showed CCR9 and its ligand CCL25 is co-expressed in ovarian cancer carcinomas depending on the different stages.

\section{Discussion}

Ovarian cancer is the most lethal gynecological malignancy and the fifth leading cause of cancer-related deaths among women in the United States. Late diagnosis and metastasis are major causes for this high mortality rate (14). Chemokines have been shown to play important roles in organ-specific homing of cancer cells to distant organs $(5,15)$. CCL25-CCR9 interactions are key to leukocyte homing to the small bowel $(16,17)$, a common and fatal site of ovarian cancer metastasis. CCL25-CCR9 interactions have been previously implicated in the progression of melanoma and prostate cancers $(12,18)$. Other studies concluded CCR9 is highly expressed by melanoma cells and all melanoma cells isolated from small intestine metastases (19). In this regard, high levels of CCL25 in the gut mucosa and expression of CCR9 by ovarian cancer cell lines suggest CCL25-CCR9 interactions might play a significant role in the mucosal homing of ovarian cancer cells.

Ovarian tumors display histological heterogeneity (20). The histological classification of ovarian tumors by the World Health Organization (WHO) is based on the histogenesis of 


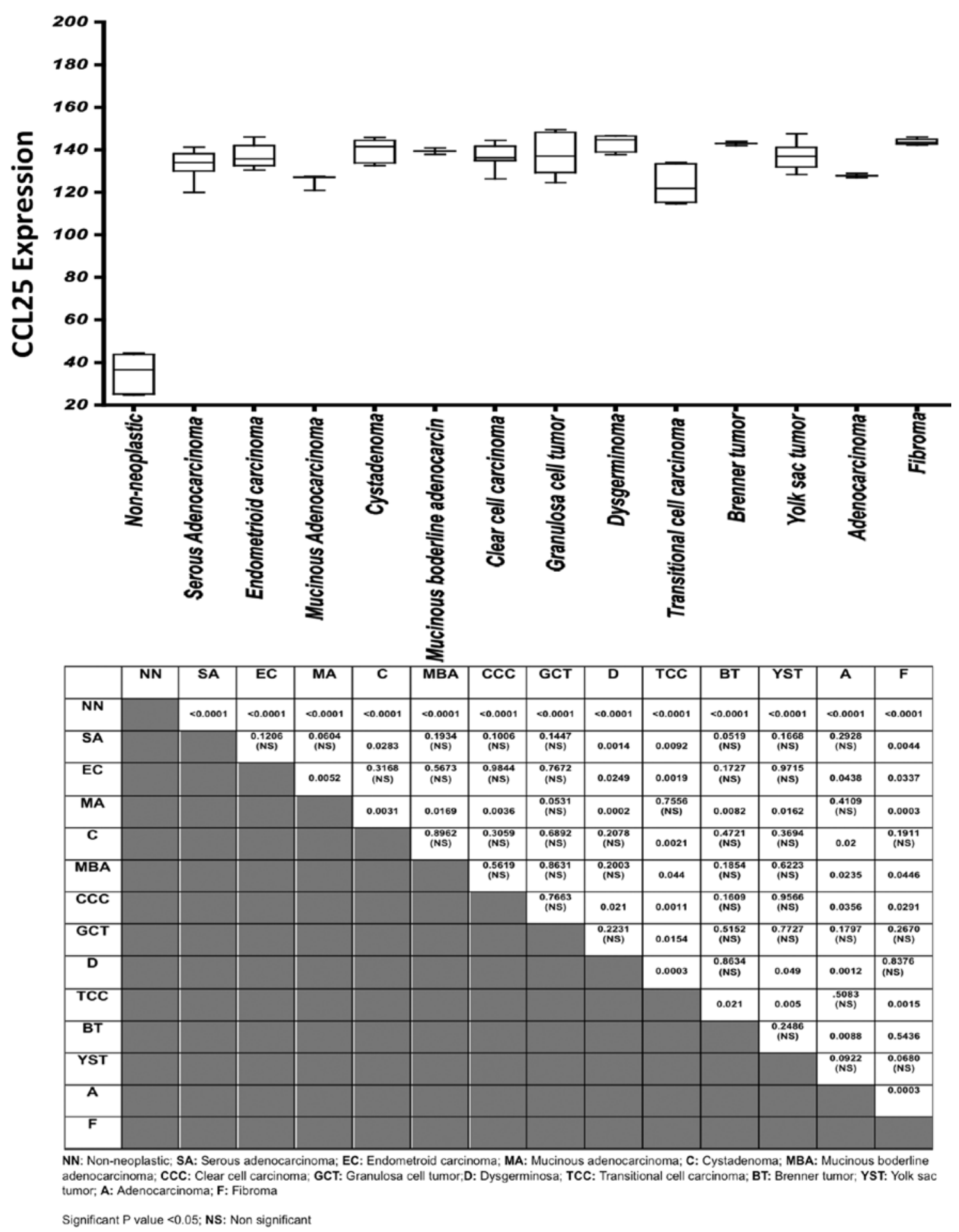

Figure 4. Analysis of CCL25 expressed by the ovarian cancer tissue. CCL25 expression was analyzed and presented as a modified box plot. Lower, middle and upper lines, respectively, in the box represent the first quartile (Q1), median (Q2) and third quartile (Q3). Upper (T) and lower (I) whiskers are represented by median \pm 1.5 (Q3-Q1). Significant differences from non -neoplastic are indicated in table with their respective p-value to show significant differences between serous adenocarcinoma, serous papillary cystadenoma, endometrioid adenocarcinoma, mucinous adenocarcinoma, Cystadenoma, mucinous boderline adenocarcinoma, clear cell carcinoma, granulosa cell tumor, dysgerminoma, transitional cell carcinoma, Brenner tumor, yolk sac tumor, adenocarcinoma and fibroma.

the normal ovary (20). The histogenetic classification categorizes ovarian neoplasms with regard to their derivation from coelomic surface epithelium, germ cells, and mesenchyme (the stroma and the sex cord). The majority of malignant ovarian tumors are epithelial. They can be further grouped into histological types as follows: serous, mucinous, endometrioid, clear cell, transitional cell tumors (Brenner tumors), mixed epithelial tumor and others. Efforts at early detection and new therapeutic approaches to reduce mortality have been largely unsuccessful, because the origin and pathogenesis of epithelial ovarian cancer are poorly understood. Despite numerous studies that have carefully scrutinized the ovaries for precursor lesions, none 


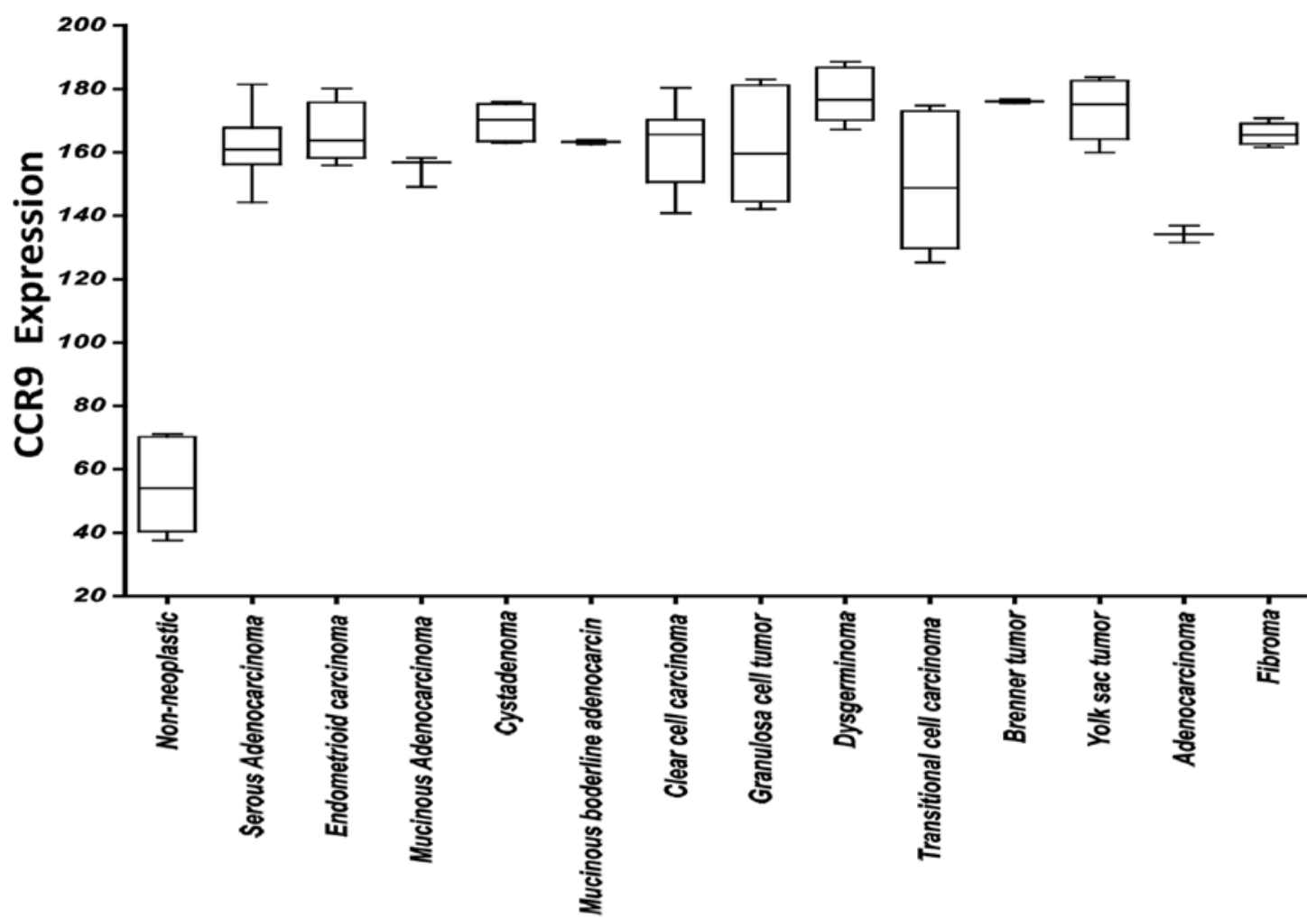

\begin{tabular}{|c|c|c|c|c|c|c|c|c|c|c|c|c|c|c|}
\hline & NN & SA & $\overline{E C}$ & $\overline{M A}$ & $\bar{C}$ & MBA & CCC & $\overline{\text { GCT }}$ & $\bar{D}$ & TCC & BT & YST & $\bar{A}$ & $\bar{F}$ \\
\hline $\mathrm{NN}$ & & $<0.0001$ & $<0.00001$ & $<0.0001$ & $<0.0001$ & $<0.0001$ & $<0.0001$ & $<0.0001$ & $<0.0001$ & $<0.0001$ & $<0.0001$ & $<0,001$ & $<0,0001$ & $<0000$ \\
\hline SA & & & 0.2374 & $\begin{array}{c}0.3044 \\
\text { NS }\end{array}$ & 0.0823 & $\begin{array}{l}0.8114 \\
\text { (Ns }\end{array}$ & 0.8861 & 0.9564 & 0.0023 & $\begin{array}{l}0.0998 \\
\text { (N) }\end{array}$ & 0.0709 & 0.0123 & 00025 & 0.4221 \\
\hline EC & & & & $\begin{array}{l}0.0614 \\
\text { (NS) }\end{array}$ & $\begin{array}{l}0.43922 \\
\text { (NS) }\end{array}$ & $\begin{array}{l}0.6338 \\
\text { o. (NS) } \\
\text { (NS) }\end{array}$ & $\begin{array}{l}0.3916 \\
\text { (NS) } \\
\text { (NS) }\end{array}$ & $\begin{array}{l}0.55097 \\
\text { (NS) } \\
\text { (NS) }\end{array}$ & 0.0251 & 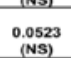 & $\begin{array}{l}0.1758 \\
\text { (NS) } \\
\text { (NS) }\end{array}$ & $\begin{array}{l}0.1449 \\
\text { (NS) } \\
\text { (NS }\end{array}$ & 0.0009 & $\begin{array}{l}0.9109 \\
\text { (NS) }\end{array}$ \\
\hline MA & & & & & 0.0059 & $\begin{array}{l}0.1072 \\
\text { (NS) }\end{array}$ & $\begin{array}{c}0.3453 \\
\text { (NS) }\end{array}$ & $\begin{array}{c}0.53833 \\
\text { (NS) }\end{array}$ & 0.0026 & $\begin{array}{c}0.7341 \\
\text { (NS) }\end{array}$ & 0.0106 & 0.0136 & 0.0163 & 19 \\
\hline C & & & & & & $\begin{array}{c}0.1733 \\
(\mathrm{NS})\end{array}$ & $\begin{array}{c}0.1739 \\
\text { (NS) }\end{array}$ & $\begin{array}{l}0.3209 \\
\text { (NS) }\end{array}$ & $\begin{array}{c}0.06652 \\
\text { (NS) }\end{array}$ & 0.0451 & $\begin{array}{c}0.1758 \\
\text { (NS) }\end{array}$ & $\begin{array}{l}0.4252 \\
\text { (NS) } \\
\end{array}$ & 0.0002 & $\begin{array}{l}0.2741 \\
\text { (NS) }\end{array}$ \\
\hline MBA & & & & & & & $\begin{array}{c}0.8958 \\
\text { (NS) }\end{array}$ & $\overbrace{\substack{0.9127 \\
\text { (NS) }}}$ & 0.0473 & $\begin{array}{c}0.4205 \\
\text { (NS) } \\
\text { (NS) }\end{array}$ & 0.0052 & $\begin{array}{l}0.2012 \\
\text { (NS) }\end{array}$ & 0.0088 & $\begin{array}{c}0.4415 \\
\text { (NS) }\end{array}$ \\
\hline CCC & & & & & & & & $\begin{array}{c}0.9673 \\
\text { (NS) }\end{array}$ & 0.0139 & $\begin{array}{c}0.1676 \\
\text { (NS) }\end{array}$ & $\begin{array}{c}0.1485 \\
\text { (NS) }\end{array}$ & 0.0488 & 0.0115 & $\begin{array}{l}0.5601 \\
\text { (NS) }\end{array}$ \\
\hline GCT & & & & & & & & & $\begin{array}{c}0.0706 \\
\text { (NS) }\end{array}$ & $\begin{array}{c}0.3259 \\
\text { (NS) }\end{array}$ & $\begin{array}{c}0.3205 \\
\text { (NS) }\end{array}$ & 0.1412 & $\begin{array}{c}0.0843 \\
\text { (NS) }\end{array}$ & 0.0662 \\
\hline D & & & & & & & & & & 0.0104 & $\begin{array}{c}0.7817 \\
\text { (NS) }\end{array}$ & $\begin{array}{l}0.3901 \\
\text { (NS) }\end{array}$ & 0.0003 & 0.0242 \\
\hline TCC & & & & & & & & & & & $\begin{array}{c}0.1330 \\
\text { (NS) }\end{array}$ & 0.0143 & $\begin{array}{c}0.3117 \\
\text { (NS) }\end{array}$ & $\begin{array}{c}0.1712 \\
\text { (NS) }\end{array}$ \\
\hline BT & & & & & & & & & & & & $\begin{array}{c}0.7235 \\
\text { (NS) }\end{array}$ & 0.0042 & 0.0286 \\
\hline YST & & & & & & & & & & & & & 0.0007 & 0.1794 \\
\hline A & & & & & & & & & & & & & & \\
\hline & & & & & & & & & & & & & & \\
\hline
\end{tabular}

NN: Non-neoplastic; SA: Serous adenocarcinoma; EC: Endometroid carcinoma; MA: Mucinous adenocarcinoma; C: Cystadenoma; MBA: Mucinous boderline adenocarcinoma; CCC: Clear cell carcinoma; GCT: Granulosa cell tumor;D: Dysgerminosa; TCC: Transitional cell carcinoma; BT: Brenner tumor; YST: Yolk sac tumor; A: Adenocarcinoma; F: Fibroma

Significant $P$ value $<0.05 ;$ NS: Non significant

Figure 5. Analysis of CCR9 expressed by the ovarian cancer tissue. CCR9 expression was analyzed and presented as a modified box plot. Lower, middle and upper lines, respectively, in the box represent the first quartile (Q1), Median (Q2) and third quartile (Q3). Upper (T) and lower (I) whiskers are represented by median \pm 1.5 (Q3-Q1). Significant differences from non -neoplastic are indicated in table with their respective p-value to show significant differences between serous adenocarcinoma, serous papillary cystadenoma, endometrioid adenocarcinoma, mucinous adenocarcinoma, Cystadenoma, mucinous boderline adenocarcinoma, clear cell carcinoma, granulosa cell tumor, dysgerminoma, transitional cell carcinoma, Brenner tumor, yolk sac tumor, adenocarcinoma, and fibroma.

have been found. This has led to the proposal that ovarian cancer develops de novo. Studies have shown that epithelial ovarian cancer is not a single disease but is composed of a diverse group of tumors that can be classified based on distinctive morphologic and molecular genetic features. One group of tumors, designated type I, is composed of low-grade serous, low-grade endometrioid, clear cell, mucinous and transitional (Brenner) carcinomas. These tumors generally behave in an indolent fashion, are confined to the ovary at presentation and, as a group, are relatively genetically stable. They lack mutations of TP53, but each histologic type exhibits a distinctive molecular genetic profile. Moreover, the carcinomas exhibit a shared 
lineage with the corresponding benign cystic neoplasm, often through an intermediate (borderline tumor) step, supporting the morphologic continuum of tumor progression (21).

In this study, we used immunohistochemical staining to examine CCR9 and CCL25 expression in non-neoplastic and epithelial ovarian cancer tissue. We failed to detect CCR9 and CCL25 expression in non-neoplastic ovarian surface epithelium, but they were co-expressed in the follicular cells of ovarian cortex. Ovarian cancer cells mainly showed cytoplasmic CCR9 staining. We have previously shown that human prostate tumor cells express CCR9 $(12,13)$. The specific ligand of this receptor is CCL25, previously it was reported that CCR9 is expressed on human melanoma cells and participates in the enhanced motility of melanoma cells and is likely a 'homing receptor' for melanoma to the small bowel $(18,19)$. The ligand for CCR9, CCL25, is strongly expressed in the small bowel and the thymus $(11,22)$. Tumors of surface epithelial origin constitute about two thirds of all ovarian neoplasms and an even greater proportion of ovarian malignant neoplasms. They occur predominantly in adults, with the malignant forms generally appearing later in life. Serous neoplasms are the most common neoplasms. Macroscopically, serous cystadenoma is often a unilocular cyst, with a smooth surface and filled with serous fluid, and sometimes consists of multilocular cysts. Adenofibroma is a predominantly solid fibrous tumor. Serous carcinoma expresses CCR9 and CCL25 (Fig. 2); it exhibits a mixture of cystic, papillary, and solid growth patterns. The carcinoma often invades through the ovarian capsule and grows on the surface of the ovary. Foci of necrosis and hemorrhage are common in serous carcinoma. Serous adenoma is lined by tubal-type epithelium. The tubal-type epithelium is composed of tall ciliated and non-ciliated cuboidal cells with elongated nuclei, and intercalated and clear cells are fewer. In serous adenofibroma, the stroma is the dominant component of the neoplasm. A serous borderline tumor is lined by tubal-type epithelium as is a benign serous tumor, but with fewer ciliated cells and some evidence of proliferative activity. The cells are stratified into several layers, forming tufts. Complex papillary and glandular growth patterns are characteristic. The tumor cells exhibit mild to marked nuclear atypia, and mitotic figures are present, but neither of these features is as pronounced as in serous carcinoma. Obvious stromal invasion is not identified. However, peritoneal implants of this tumor are rarely identified (23). Serous carcinoma is composed in part of papillae lined by stratified cells of serous type. More extensive cellular budding, more confluent cellular growth, and usually greater nuclear atypia than serous borderline tumors characterize serous carcinomas by obvious stromal invasion (Fig. 2). The detection of CCR9 and CCL25 protein expression should be useful for the diagnosis and prognosis of ovarian serous cancers, and CCL25 might be a useful molecular target for ovarian cancer therapy.

Mucinous cystadenoma is one of the most common ovarian neoplasms, we have shown it expresses CCR9 and CCL25 and shows significant difference in comparison to non-neoplastic type. Mucinous adenocarcinomas are predominantly solid. Necrosis and hemorrhage are more common in invasive than borderline and benign mucinous tumors. Most of the neoplastic cells contain abundant cytoplasmic mucin, but some have mucin-free and eosinophilic cytoplasm. The stroma is diffusely infiltrated with neutrophils. This tumor is often associated with endometriosis. In contrast, intestinal type mucinous borderline tumors either lack papillae or have branching papillae. The cysts and papillae are lined by atypical epithelium that contains variable numbers of goblet cells and other intestinal cell types (24). Similarly, endometrioid tumors also express CCR9 as well as CCL25; histologically resembling adenocarcinoma of the endometrium (25). These tumors are solid masses with a soft, firm, or fibrous consistency. They are often predominantly cystic and contain soft or firm solid nodular masses. These cysts are usually filled with chocolatecolored fluid. Because most patients with mucinous adenocarcinoma are in the early stage (26), these patients show a better prognosis than those with other histological types (27). It is known that the majority of mucinous adenocarcinoma is either well- or moderately- differentiated and this contributes to the low risk of relapse for FIGO stage I tumors (27). We also found that patients in the early stage showed a good outcome. On the other hand, patients with advanced mucinous adenocarcinoma had a poorer outcome, which is well recognized, supporting the findings of Winter et al (28). There was no significant difference in survival between mucinous invasive adenocarcinoma and serous adenocarcinoma in patients with optimal operation. In contrast, patients with suboptimal operation showed a significantly worse prognosis than those with serous adenocarcinoma. CCR9-CCL25 expression was strongly associated with endometroid histological subtype. In ovarian cancer, however, CCR9 staining seems to be of prognostic importance with respect to staining in univariate analysis.

We have shown that clear cell tumors, comprise $5-10 \%$ of all ovarian cancers in Western countries (29) and expressed higher CCL25. Microscopically, clear cell carcinoma contains clear cells and hobnail cells. Clear cells are typically polygonal, have distinct cell membranes, and contain abundant clear cytoplasm and eccentric nuclei (Fig. 2). The clear cells grow as tubular, papillary, solid, or frequently mixed types. The hobnail cells are characterized by apical nuclei that protrude into the lumen of tubules and cysts beyond the cytoplasmic limits of the cells. Multiple complex papillae are often encountered. The hyaline condensations of basement membrane materials are seen, particularly in the core of papillae. The transition between endometriosis and clear cell carcinoma is often observed.

Nests and columns of epithelial cells characterize Brenner tumors, majority are of transitional type, resembling urothelial cells. These tumor nests are distributed within a predominant fibrous stroma. Cells filled with mucin are present in varying amounts in one-third of the cases. Malignant transitional cell tumors (Brenner tumors) resemble a high-grade transitional cell carcinoma of the bladder (30).

We demonstrated that clear cell and endometrioid carcinomas express significantly higher CCR9 and CCL25 than other tumors; these carcinomas tend to remain confined to the ovary, and approximately half the cases are associated with endometriosis. Clear cell and endometrioid carcinomas may arise from the ovarian endometriosis, and the ovary is primary site of these tumors. Therefore, clear cell carcinomas in extra ovarian extension may have an aggressive clinical course. 
In contrast, serous carcinomas generally have extensive peritoneal implantation and ascites; spread above the pelvic floor is evident at operation in more than half the cases. Lymph node metastasis and pulmonary involvement are frequent, particularly in the late stages of the disease; hematogenous metastases are occasionally encountered. These tumors arise from the surface epithelium, which is basically similar to peritoneal surface epithelium, most often via neoplastic transformation of a surface epithelial inclusion cyst. Clear cell and endometrioid carcinomas may be unique histological types compared with serous carcinomas in respect to stage distribution and association with endometriosis.

We provide the first evidence that ovarian cancer cells and patient tissue express functional CCR9 and CCL25. Expression of the receptor and its natural ligand with in primary tumor tissue suggest that this chemokine-receptor axis may be involved in ovarian cancer progression.

\section{Acknowledgements}

The content of this manuscript benefited from many fruitful conversations with members of the Morehouse School of Medicine, the University of Alabama at Birmingham. This study was supported by the National Institute of Health grant (RR03034) and also supported by the Research Centers in Minority Institutions Program (RCMI) funded Flow Cytometry and Cell Sorting Core at Morehouse School of Medicine, Atlanta.

\section{References}

1. Jemal A, Siegel R, Ward E, Hao Y, Xu J and Thun MJ: Cancer statistics, 2009. CA Cancer J Clin 59: 225-249, 2009.

2. Liotta LA: An attractive force in metastasis. Nature 410: 24-25, 2001.

3. Nicolson GL: Paracrine and autocrine growth mechanisms in tumor metastasis to specific sites with particular emphasis on brain and lung metastasis. Cancer Metastasis Rev 12: 325-343, 1993.

4. Zlotnik A and Yoshie O: Chemokines: a new classification system and their role in immunity. Immunity 12: 121-127, 2000.

5. Muller A, Homey B, Soto $\mathrm{H}$, et al: Involvement of chemokine receptors in breast cancer metastasis. Nature 410: 50-56, 2001.

6. Wurbel MA, Malissen M, Guy-Grand D, et al: Mice lacking the CCR9 CC-chemokine receptor show a mild impairment of early $\mathrm{T}$ - and B-cell development and a reduction in T-cell receptor gammadelta(+) gut intraepithelial lymphocytes. Blood 98: 2626-2632, 2001.

7. Zabel BA, Agace WW, Campbell JJ, et al: Human G proteincoupled receptor GPR-9-6/CC chemokine receptor 9 is selectively expressed on intestinal homing $\mathrm{T}$ lymphocytes, mucosal lymphocytes, and thymocytes and is required for thymus-expressed chemokine-mediated chemotaxis. J Exp Med 190: 1241-1256, 1999.

8. Kunkel EJ, Campbell JJ, Haraldsen G, et al: Lymphocyte CC chemokine receptor 9 and epithelial thymus-expressed chemokine (TECK) expression distinguish the small intestinal immune compartment: epithelial expression of tissue-specific chemokines as an organizing principle in regional immunity. J Exp Med 192: $761-768,2000$.
9. Johansson-Lindbom B, Svensson M, Pabst O, et al: Functional specialization of gut $\mathrm{CD}_{103^{+}}$dendritic cells in the regulation of tissue-selective T cell homing. J Exp Med 202: 1063-1073, 2005.

10. Autschbach F, Funke B, Katzenmeier M and Gassler N: Expression of chemokine receptors in normal and inflamed human intestine, tonsil, and liver - an immunohistochemical analysis with new monoclonal antibodies from the 8th international workshop and conference on human leucocyte differentiation antigens. Cell Immunol 236: 110-114, 2005.

11. Papadakis KA, Prehn J, Moreno ST, et al: CCR9-positive lymphocytes and thymus-expressed chemokine distinguish small bowel from colonic Crohn's disease. Gastroenterology 121: 246-254, 2001.

12. Singh S, Singh UP, Stiles JK, Grizzle WE and Lillard JW: Expression and functional role of CCR9 in prostate cancer cell migration and invasion. Clin Cancer Res 10: 8743-8750, 2004.

13. Sharma PK, Singh R, Novakovic KR, Eaton JW, Grizzle WE and Singh S: CCR9-mediates PI3K/AKT dependent anti-apoptotic signals in prostate cancer cells and inhibition of CCR9-CCL25 interaction enhances the cytotoxic effects of etoposide. Int J Cancer 127: 2020-2030, 2010.

14. Cvetkovic D: Early events in ovarian oncogenesis. Reprod Biol Endocrinol 1: 68, 2003.

15. Strieter RM: Chemokines: Not just leukocyte chemoattractants in the promotion of cancer. Nat Immunol 2: 285, 2001.

16. Koenecke C and Forster R: CCR9 and inflammatory bowel disease. Expert Opin Ther Targets 13: 297-306, 2009.

17. Kabelitz D and Wesch D: Features and functions of gamma delta T lymphocytes: focus on chemokines and their receptors. Crit Rev Immunol 23: 339-370, 2003.

18. Hwang ST: Chemokine receptors in melanoma: CCR9 has a potential role in metastasis to the small bowel. J Invest Dermatol 122: xiv-xv, 2004.

19. Letsch A, Keilholz U, Schadendorf D, et al: Functional CCR9 expression is associated with small intestinal metastasis. J Invest Dermatol 122: 685-690, 2004.

20. Selvaggi SM: Tumors of the ovary, maldeveloped gonads, fallopian tube, and broad ligament. Arch Pathol Lab Med 124: 477, 2000.

21. Kurman RJ and Shih Ie M: The origin and pathogenesis of epithelial ovarian cancer: a proposed unifying theory. Am J Surg Pathol 34: 433-443, 2010.

22. Mora JR, Bono MR, Manjunath N, et al: Selective imprinting of gut-homing T cells by Peyer's patch dendritic cells. Nature 424: 88-93, 2003.

23. Bell DA, Weinstock MA and Scully RE: Peritoneal implants of ovarian serous borderline tumors. Histologic features and prognosis. Cancer 62: 2212-2222, 1988.

24. Acs G: Serous and mucinous borderline (low malignant potential) tumors of the ovary. Am J Clin Pathol 123 (Suppl): S13-S57, 2005.

25. Tsuruchi N, Kaku T, Kinoshita H, et al: Ovarian mucinous cystadenocarcinoma with sarcoma-appearing mural nodule of anaplastic carcinoma. Gynecol Oncol 50: 259-263, 1993.

26. Malkasian GD Jr, Melton LJ, III, O'Brien PC and Greene MH: Prognostic significance of histologic classification and grading of epithelial malignancies of the ovary. Am J Obstet Gynecol 149: 274-284, 1984.

27. Harrison ML, Jameson C and Gore ME: Mucinous ovarian cancer. Int J Gynecol Cancer 18: 209-214, 2008.

28. Winter WE III, Maxwell GL, Tian C, et al: Prognostic factors for stage III epithelial ovarian cancer: a Gynecologic Oncology Group Study. J Clin Oncol 25: 3621-3627, 2007.

29. Fujiu K, Miyamoto H, Hashimoto S, et al: A case of diaphragmatic clear cell carcinoma in a patient with a medical history of ovarian endometriosis. Int J Clin Oncol 15: 489-492, 2010.

30. Silva EG, Robey-Cafferty SS, Smith TL and Gershenson DM: Ovarian carcinomas with transitional cell carcinoma pattern. Am J Clin Pathol 93: 457-465, 1990. 